\title{
CALORIC MEASURE NULL SETS
}

\author{
Neil A. WATson
}

(Received 14 September, 2020)

\begin{abstract}
We give a systematic treatment of caloric measure null sets on the essential boundary $\partial_{e} E$ of an arbitrary open set $E$ in $\mathbb{R}^{n+1}$. We discuss two characterisations of such sets and present some basic properties. We investigate the dependence of caloric measure null sets on the open set $E$. Thus, if $D$ is an open subset of $E$ and $Z \subseteq \partial_{e} E \cap \partial_{e} D$, we show that $Z$ is caloric measure null for $D$ if it is caloric measure null for $E$. We also give conditions on $E$ and $Z$ which imply that the reverse implication is true. We know from [10] that any polar subset of $\partial_{e} D$ is caloric measure null for $D$, but the reverse implication is not generally true. In our final result we show that, for subsets of a certain component of $\partial_{e} D$, caloric measure null sets are necessarily polar.
\end{abstract}

\section{Introduction}

Caloric measure is sometimes called parabolic measure, sometimes harmonic measure for the heat equation. Its null sets have been studied by several authors for particular boundaries, for example in $[\mathbf{3}, \mathbf{4}, \mathbf{5}, \mathbf{6 ,} \mathbf{7}, \mathbf{1 4}, \mathbf{1 5}]$. However, to my knowledge they have never been given a systematic treatment for arbitrary open sets. In this paper we give such a treatment, partly guided by the treatments for Laplace's equation given in $[\mathbf{1}, \mathbf{2}]$, but also including results that have no known analogue in the classical case.

Our terminology will follow $[\mathbf{1 1}]$, where further details can be found. We work in $\mathbb{R}^{n+1}=\left\{(x, t): x \in \mathbb{R}^{n}, t \in \mathbb{R}\right\}$, and denote a typical point by $p$ or $(x, t)$ as convenient. The characteristic function of a set $A$ is denoted by $\chi_{A}$.

Given an open set $E$ in $\mathbb{R}^{n+1}$, a function $u \in C^{2,1}(E)$ that satisfies the standard heat equation $\sum_{i=1}^{n}\left(\partial^{2} u / \partial x_{i}^{2}\right)-(\partial u / \partial t)=0$ on $E$ is called a temperature. If

$$
W(x, t)= \begin{cases}(4 \pi t)^{-\frac{n}{2}} \exp \left(-\frac{|x|^{2}}{4 t}\right) & \text { if } t>0, \\ 0 & \text { if } t \leq 0 .\end{cases}
$$

then $W$ is a temperature on $\mathbb{R}^{n+1} \backslash\{0\}$. For any point $\left(x_{0}, t_{0}\right) \in \mathbb{R}^{n+1}$ and any positive number $c$, the set

$$
\Omega\left(x_{0}, t_{0} ; c\right)=\left\{(y, s) \in \mathbb{R}^{n+1}: W\left(x_{0}-y, t_{0}-s\right)>(4 \pi c)^{-\frac{n}{2}}\right\}
$$

is called the heat ball with centre $\left(x_{0}, t_{0}\right)$ and radius $c$. Temperatures can be characterized in terms of mean values over heat balls, in that a function $u \in C^{2,1}(E)$

2010 Mathematics Subject Classification Primary: 35K05; Secondary: 31B05, 31B10, 31B15, 31B20.

Key words and phrases: Caloric measure, Dirichlet problem, temperature, polar set. 
is a temperature if and only if

$$
u\left(x_{0}, t_{0}\right)=(4 \pi c)^{-\frac{n}{2}} \iint_{\Omega\left(x_{0}, t_{0} ; c\right)} \frac{\left|x_{0}-x\right|^{2}}{4\left(t_{0}-t\right)^{2}} u(x, t) d x d t
$$

whenever $\bar{\Omega}\left(x_{0}, t_{0} ; c\right) \subseteq E$.

An extended real-valued function $v$ on $E$ is called a supertemperature on $E$ if it satisfies the following four conditions:

$\left(\delta_{1}\right):-\infty<v(p) \leq+\infty$ for all $p \in E$.

$\left(\delta_{2}\right): v$ is lower semicontinuous on $E$.

$\left(\delta_{3}\right): v$ is finite on a dense subset of $E$.

$\left(\delta_{4}\right)$ : Given any point $\left(x_{0}, t_{0}\right) \in E$ and positive number $\epsilon$, there is a positive number $c<\epsilon$ such that the closed heat ball $\bar{\Omega}\left(x_{0}, t_{0} ; c\right) \subseteq E$ and the inequality

holds.

$$
v\left(x_{0}, t_{0}\right) \geq(4 \pi c)^{-\frac{n}{2}} \iint_{\Omega\left(x_{0}, t_{0} ; c\right)} \frac{\left|x_{0}-x\right|^{2}}{4\left(t_{0}-t\right)^{2}} u(x, t) d x d t
$$

An extended real-valued function $v$ on $E$ is called a hypertemperature on $E$ if it satisfies the conditions $\left(\delta_{1}\right),\left(\delta_{2}\right)$ and $\left(\delta_{4}\right)$ above.

We require a classification of the boundary points of an arbitrary open set $E$. Here, and below, we use the following notations for the upper and lower half-balls. Given a point $p_{0}=\left(x_{0}, t_{0}\right) \in \mathbb{R}^{n+1}$ and $r>0$, we denote by $H\left(p_{0}, r\right)$ the open lower half-ball $\left\{(x, t):\left|x-x_{0}\right|^{2}+\left(t-t_{0}\right)^{2}<r^{2}, t<t_{0}\right\}$, and by $H^{*}\left(p_{0}, r\right)$ the open upper half-ball $\left\{(x, t):\left|x-x_{0}\right|^{2}+\left(t-t_{0}\right)^{2}<r^{2}, t>t_{0}\right\}$.

Definitions. Let $q$ be a boundary point of the open set $E$. We call $q$ a normal boundary point if either

(a) $q$ is the point at infinity, or

(b) $q \in \mathbb{R}^{n+1}$, and for every $r>0$ we have $H(q, r) \backslash E \neq \emptyset$.

Otherwise, we call $q$ an abnormal boundary point; in this case, there is some $r_{0}>0$ such that $H\left(q, r_{0}\right) \subseteq E$. The abnormal boundary points are of two kinds, according to whether they can be approached from above by points in $E$. If there is some $r_{1}<r_{0}$ such that $H^{*}\left(q, r_{1}\right) \cap E=\emptyset$, then $q$ is called a singular boundary point. On the other hand if, for every $r<r_{0}$, we have $H^{*}(q, r) \cap E \neq \emptyset$, then $q$ is called a semi-singular boundary point.

The set of all normal boundary points of $E$ is denoted by $\partial_{n} E$, that of all abnormal points by $\partial_{a} E$, that of all singular points by $\partial_{s} E$, and that of all semisingular points by $\partial_{s s} E$. Thus $\partial E=\partial_{n} E \cup \partial_{a} E$ and $\partial_{a} E=\partial_{s} E \cup \partial_{s s} E$. The essential boundary $\partial_{e} E$ is defined by

$$
\partial_{e} E=\partial_{n} E \cup \partial_{s s} E=\partial E \backslash \partial_{s} E .
$$

We also use the concept of an abnormal boundary point relative to the adjoint equation $\sum_{i=1}^{n}\left(\partial^{2} u / \partial x_{i}^{2}\right)+(\partial u / \partial t)=0$. If $q$ is such a point, then there is some $r_{0}>0$ such that $H^{*}\left(q, r_{0}\right) \subseteq E$. The set of all such points is denoted by $\partial_{a}^{*} E$.

Let $f$ be an extended real-valued function defined on $\partial_{e} E$. For any lower bounded hypertemperature $v$ on $E$, we put $v$ in the class $\mathfrak{U}_{f}^{E}$ if and only if both

$$
\liminf _{p \rightarrow q} v(p) \geq f(q) \text { for all } q \in \partial_{n} E,
$$


and

$$
\liminf _{p \rightarrow q+} v(p) \geq f(q) \quad \text { for all } q \in \partial_{s s} E
$$

where the notation $\liminf _{p \rightarrow q+} v(p)$ means $\liminf _{(x, t) \rightarrow(y, s+)} v(x, t)$. We then put $U_{f}^{E}=\inf \left\{v: v \in \mathfrak{U}_{f}^{E}\right\}$, and call it the upper solution for $f$ on $E$. The lower solution $L_{f}^{E}$ can be defined by the formula $L_{f}^{E}=-U_{-f}^{E}$. This is the same as in $[\mathbf{9 , 1 1}]$.

If $L_{f}^{E}=U_{f}^{E}$ and is a temperature on $E$, we denote it by $S_{f}^{E}$, call it the $P W B$ solution for $f$ on $E$, and say that $f$ is resolutive for $E$. Every function $f \in C\left(\partial_{e} E\right)$ is resolutive, a result first proved in $[\mathbf{9}]$. For any point $p \in E$ there is a unique nonnegative Borel measure $\mu_{p}^{E}$ on $\partial_{e} E$ such that $S_{f}^{E}(p)=\int_{\partial_{e} E} f d \mu_{p}^{E}$ holds for every $f \in C\left(\partial_{e} E\right)$. The completion of this measure is called the caloric measure relative to $E$ and $p$; it is also denoted by $\mu_{p}^{E}$. If $f$ is resolutive for $E$, then $S_{f}^{E}$ has the representation $S_{f}^{E}(p)=\int_{\partial_{e} E} f d \mu_{p}^{E}$ for all $p \in E$. A point $q \in \partial_{n} E$ is called regular if $\lim _{p \rightarrow q} S_{f}^{E}(p)=f(q)$ for all $f \in C\left(\partial_{e} E\right)$. A point $q \in \partial_{s s} E$ is called regular if $\lim _{p \rightarrow q+} S_{f}^{E}(p)=f(q)$ for all $f \in C\left(\partial_{e} E\right)$. The set $E$ is called regular if every point of $\partial_{e} E$ is regular, and quasi-regular if every point of $\partial_{e} E$ outside some polar set is regular.

Given a point $p_{0} \in E$, we denote by $\Lambda\left(p_{0} ; E\right)$ the set of points $q \in E$ that are lower than $p_{0}$ relative to $E$, in the sense that there is a polygonal path $\gamma \subseteq E$ joining $p_{0}$ to $q$ along which the temporal variable $t$ is strictly decreasing. We also denote by $\Lambda^{*}\left(p_{0} ; E\right)$ the set of points $q \in E$ for which there is a polygonal path $\gamma \subseteq E$ joining $p_{0}$ to $q$ along which $t$ is strictly increasing. Clearly $p \in \Lambda(q ; E)$ if and only if $q \in \Lambda^{*}(p ; E)$.

We recall from $[\mathbf{1 0}]$ that a subset $Z$ of $\partial_{e} E$ is a caloric measure null set for $E$ if $\mu_{p}^{E}(Z)=0$ for all $p \in E$. An equivalent condition is that $U_{\chi_{Z}}^{E}=0$ on $E$, and $\chi_{Z}$ is resolutive.

In Section 2 we amend the characterization of caloric measure null sets given in [10], and give a new characterization which relates the null sets of $E$ to those of $\Lambda(q ; E)$ for points $q \in E$. We also present some basic properties of caloric measure null sets. All of these results are analogues of known results for harmonic measure null sets.

In Section 3 we look at the dependence of caloric measure null sets on the set $E$. Let $D$ and $E$ be open sets such that $D \subseteq E$. If $q \in D$, and $Z$ is a subset of $\partial_{e} E \cap \partial_{e} D$ which is $\mu_{q}^{E}$-measurable, we know from [12, Theorem 10] that $Z$ is also $\mu_{q}^{D}$-measurable with $\mu_{q}^{D}(Z) \leq \mu_{q}^{E}(Z)$. It is easy to deduce from this that $Z$ is caloric measure null for $D$ if it is caloric measure null for $E$. We prove, under certain conditions on $E$ and $Z$, that the reverse implication is also true. These results are also analogues of known results for harmonic measure null sets.

Although a polar subset of $\partial_{e} D$ is always caloric measure null for $D$, the opposite implication is only rarely true. Indeed, as shown in [13, Example 5], a caloric measure null set can have positive $(n+1)$-dimensional Lebesgue measure. In Section 4 we show that a subset $Z$ of $\partial_{a}^{*} D$ is caloric measure null for $D$ if and only if it is polar. Note that if $q \in \partial_{a}^{*} D$ there is an upper half-ball $H^{*}(q, r) \subseteq D$, so that $q \notin \partial_{s} D$ and hence $q \in \partial_{e} D$. 


\section{Characterizations and General Properties}

We need the characterization of caloric measure null sets given in $[\mathbf{1 0}$, Theorem $5.2]$. In the proof of that result, it was implicitly assumed that $\partial_{e} E$ is always equal to $\bigcup_{p \in E} \partial_{e} \Lambda(p ; E)$, which is not the case. Certainly [10, Lemma 2.9] shows that we always have $\bigcup_{p \in E} \partial_{e} \Lambda(p ; E) \subseteq \partial_{e} E$, but the reverse inclusion may not hold. This oversight is easy to fix, because the set difference is always caloric measure null for $E$, as we shall show. Here is a corrected statement of the theorem.

Theorem 1. Let $E$ be an open set.

(a) The set

$$
T=\partial_{e} E \backslash \bigcup_{p \in E} \partial_{e} \Lambda(p ; E)
$$

is caloric measure null for $E$.

(b) If $Z \subseteq \bigcup_{p \in E} \partial_{e} \Lambda(p ; E)$, then $Z$ is caloric measure null for $E$ if and only if the following condition is satisfied.

For each point $p_{0} \in E$, there is a nonnegative supertemperature $u$ on $\Lambda\left(p_{0} ; E\right)$ such that $\lim _{p \rightarrow q} u(p)=+\infty$ for all $q \in Z \cap \partial_{n} \Lambda\left(p_{0} ; E\right)$, and $\lim _{p \rightarrow q+} u(p)=+\infty$ for all $q \in Z \cap \partial_{s s} \Lambda\left(p_{0} ; E\right)$.

Proof. (a) Given any point $p \in E$, we put $\Lambda=\Lambda(p ; E)$. The function $\chi=\chi_{T}$ is identically zero on $\partial_{e} \Lambda$, so that $U_{\chi}^{\Lambda}=0$ on $\Lambda$. By $\left[\mathbf{1 0}\right.$, Lemma 3.4], $U_{\chi}^{\Lambda}$ is the restriction to $\Lambda$ of $U_{\chi}^{E}$, so that $U_{\chi}^{E}=0$ on $\Lambda$. Since $p$ is arbitrary, $U_{\chi}^{E}=0$ on $E$.

(b) See the proof of [10, Theorem 5.2].

Here is a different characterization of caloric measure null sets. This result is analogous to one for harmonic measure given in [1, Lemma 6.5.3], where $\Lambda(q ; E)$ is replaced by the component of $E$ that contains $q$, and $\partial_{e}$ by $\partial^{\infty}$.

Theorem 2. Let $E$ be an open set, and let $Z \subseteq \partial_{e} E$.

(a) If $Z$ is caloric measure null for $E$, then given any $p \in E$ and $q \in \Lambda^{*}(p ; E)$, we have

$$
\mu_{p}^{\Lambda(q ; E)}\left(Z \cap \partial_{e} \Lambda(q ; E)\right)=0
$$

(b) Conversely, if $\left\{p_{j}\right\}$ is a sequence in $E$ such that $\bigcup_{j=1}^{\infty} \Lambda\left(p_{j} ; E\right)=E$, and for each $j$ there is a point $q_{j} \in \Lambda^{*}\left(p_{j} ; E\right)$ such that

$$
\mu_{p_{j}}^{\Lambda\left(q_{j} ; E\right)}\left(Z \cap \partial_{e} \Lambda\left(q_{j} ; E\right)\right)=0,
$$

then $Z$ is a caloric measure null set for $E$.

Proof. (a) Given any points $p \in E$ and $q \in \Lambda^{*}(p ; E)$, the set $Y=Z \cap \partial_{e} \Lambda(q ; E)$ is caloric measure null for $E$ and hence $\mu_{p}^{E}$-measurable. Therefore [12, Theorem 10] shows that $Y$ is also $\mu_{p}^{\Lambda(q ; E)}$-measurable with $\mu_{p}^{\Lambda(q ; E)}(Y)=0$.

(b) For each $j$, we denote by $\chi_{j}$ the characteristic function of $Z \cap \partial_{e} \Lambda\left(q_{j} ; E\right)$. If $\mu_{p_{j}}^{\Lambda\left(q_{j} ; E\right)}\left(Z \cap \partial_{e} \Lambda\left(q_{j} ; E\right)\right)=0$, then $\int_{\partial_{e} \Lambda\left(q_{j} ; E\right)} \chi_{j} d \mu_{p_{j}}^{\Lambda\left(q_{j} ; E\right)}$ exists so that, in view of [10, Theorem 4.6(a)],

$$
U_{\chi_{j}}^{\Lambda\left(q_{j} ; E\right)}\left(p_{j}\right)=\int_{\partial_{e} \Lambda\left(q_{j} ; E\right)} \chi_{j} d \mu_{p_{j}}^{\Lambda\left(q_{j} ; E\right)}=\mu_{p_{j}}^{\Lambda\left(q_{j} ; E\right)}\left(Z \cap \partial_{e} \Lambda\left(q_{j} ; E\right)\right)=0 .
$$


Since the values of $U_{\chi_{j}}^{\Lambda\left(q_{j} ; E\right)}$ all lie in the interval $[0,1]$, it is a temperature on $\Lambda\left(p ; \Lambda\left(q_{j} ; E\right)\right)$ for all $p \in \Lambda\left(q_{j} ; E\right)$, by $\left[\mathbf{1 0}\right.$, Lemma 3.6], and hence on $\Lambda\left(q_{j} ; E\right)$ itself. It therefore follows from the minimum principle that $U_{\chi_{j}}^{\Lambda\left(q_{j} ; E\right)}=0$ on the set $\Lambda\left(p_{j} ; \Lambda\left(q_{j} ; E\right)\right)=\Lambda\left(p_{j} ; E\right)$. Since $\chi_{j}$ is the restriction to $\partial_{e} \Lambda\left(q_{j} ; E\right)$ of $\chi_{z}$, it follows from [10, Lemma 3.4] that $U_{\chi Z}^{E}=0$ on $\Lambda\left(p_{j} ; E\right)$. Our hypothesis on $\left\{p_{j}\right\}$ now implies that $U_{\chi Z}^{E}=0$ on $E$, so that $Z$ is caloric measure null for $E$.

Theorem 3. Let $E$ be an open set.

(a) If $f$ is a function on $\partial_{e} E$ such that $U_{f}^{E}<+\infty$ on $E$, then the set $Z$ of points $p$ where $f(p)=+\infty$ is caloric measure null for $E$.

(b) If $\left\{Z_{i}\right\}$ is a sequence of caloric measure null subsets of $\partial_{e} E$, then $\bigcup_{i=1}^{\infty} Z_{i}$ is also caloric measure null for $E$.

(c) If $f$ is a nonnegative function on $\partial_{e} E$ such that $U_{f}^{E}=0$ on $E$, then the set $Z$ of points $p$ where $f(p)>0$ is caloric measure null for $E$.

(d) If $Z$ is a relatively open subset of $\partial_{e} E$ that is caloric measure null for $E$, then every point of $Z$ is irregular and $Z$ is semipolar.

Proof. (a) Let $f$ and $Z$ be as in the statement. By Theorem 1(a), we may suppose that $Z \subseteq \bigcup_{p \in E} \partial_{e} \Lambda(p ; E)$, and so we can use the criterion in Theorem 1(b). Let $p_{0} \in E$. Since $U_{f}^{E}\left(p_{0}\right)<+\infty$, we can find a hypertemperature $u \in \mathfrak{U}_{f}^{E}$ such that $u\left(p_{0}\right)<+\infty$, which implies that $u$ is a supertemperature on $\Lambda\left(p_{0} ; E\right)$ by [11, Corollary 3.55]. The function $u$ is lower bounded on $E$, and satisfies both $\lim _{p \rightarrow q} u(p)=+\infty$ for all $q \in Z \cap \partial_{n} E$ and $\lim _{p \rightarrow q+} u(p)=+\infty$ for all $q \in$ $Z \cap \partial_{s s} E$. We put $v=u-\inf _{E} u$ on $E$, so that $v$ is a nonnegative supertemperature on $\Lambda\left(p_{0} ; E\right)$. We also put $\Lambda=\Lambda\left(p_{0} ; E\right)$. By [11, Lemma 8.4], $\partial_{e} \Lambda \subseteq \partial_{e} E$ and $\partial_{s s} \Lambda \subseteq \partial_{s s} E$; but is it not in general true that $\partial_{n} \Lambda \subseteq \partial_{n} E$. Hence we immediately deduce that

$$
\lim _{p \rightarrow q+} v(p)=+\infty \text { for all } q \in Z \cap \partial_{s s} \Lambda \subseteq Z \cap \partial_{s s} E,
$$

but the corresponding result for normal boundary points needs a bit more. Clearly

$$
\lim _{p \rightarrow q} v(p)=+\infty \text { for all } q \in Z \cap \partial_{n} \Lambda \cap \partial_{n} E .
$$

On the other hand, if $q \in Z \cap \partial_{n} \Lambda \cap \partial_{s s} E$, there is an open half-ball $H(q, r) \subseteq E$ such that $H(q, r) \cap \Lambda=\emptyset$, by [11, Lemma 8.4]. Therefore

$$
\lim _{p \rightarrow q, p \in \Lambda} v(p)=\lim _{p \rightarrow q+, p \in E} v(p)=+\infty .
$$

It now follows from Theorem 1 (b) that $Z$ is caloric measure null for $E$.

(b) Let $\left\{Z_{i}\right\}$ be a sequence of caloric measure null subsets of $\partial_{e} E$. For any point $p \in E$ we have $\mu_{p}^{E}\left(Z_{i}\right)=0$ for all $i$, so that $\mu_{p}^{E}\left(\bigcup_{i=1}^{\infty} Z_{i}\right)=0$ also. Since $p$ is arbitrary, that union is caloric measure null for $E$.

(c) Let $f$ be a nonnegative function on $\partial_{e} E$ such that $U_{f}^{E}=0$ on $E$. For each $k \in \mathbb{N}$, we denote by $\chi_{k}$ the characteristic function of the set $Z_{k}$ of points $p$ where $f(p)>1 / k$. Then $0=k U_{f}^{E}=U_{k f}^{E} \geq U_{\chi_{k}}^{E} \geq 0$ on $E$, so that each $Z_{k}$ is caloric measure null for $E$. It now follows from (b) that the set $Z=\bigcup_{k=1}^{\infty} Z_{k}$ is also caloric measure null for $E$.

(d) Let $Z$ be a relatively open subset of $\partial_{e} E$ that is caloric measure null for $E$. Given any point $q \in Z$, we choose a continuous function $f: \partial_{e} E \mapsto[0,1]$ such that 
$f=0$ on $\partial_{e} E \backslash Z$ and $f(q)=1$. Then $0 \leq S_{f}^{E} \leq S_{\chi Z}^{E}=0$ on $E$ because $Z$ is caloric measure null for $E$, so that $q$ is irregular. It now follows from [11, Corollary 9.47] that $Z$ is semipolar.

The results in Theorem 3 are analogous to results for harmonic measure null sets, parts (a), (b) and (c) given in [2, p.108], part (d) in [1, Corollary 6.6.9].

The proofs of Theorems 5 and 7 require the following elementary lemma.

Lemma 4. Let $D$ be an open set, and let $Z$ be a subset of $\partial_{e} D$ which is caloric measure null for $D$. Then there is a Borel subset $B$ of $\partial_{e} D$ which contains $Z$ and is also caloric measure null for $D$.

Proof. We take a sequence $\left\{p_{j}\right\}$ of points in $D$ such that $\bigcup_{j=1}^{\infty} \Lambda\left(p_{j} ; D\right)=D$. Since $Z$ is caloric measure null for $D$, given any $j$ we have $\mu_{p_{j}}^{D}(Z)=0$ so that, since $\mu_{p_{j}}^{D}$ is a completed Borel measure, there is a Borel set $B_{j} \supseteq Z$ such that $\mu_{p_{j}}^{D}\left(B_{j}\right)=0$. We denote by $\chi_{j}$ the characteristic function of $B_{j}$. By [10, Corollary 4.7], the fact that $0 \leq L_{\chi_{j}}^{D} \leq U_{\chi_{j}}^{D} \leq 1$ implies that $\chi_{j}$ is resolutive for $D$ and

$$
S_{\chi_{j}}^{D}(p)=\int_{\partial_{e} D} \chi_{j} d \mu_{p}^{D}=\mu_{p}^{D}\left(B_{j}\right)
$$

for all $p \in D$. In particular $S_{\chi_{j}}^{D}\left(p_{j}\right)=0$, so that the temperature $S_{\chi_{j}}^{D}$ is zero on $\Lambda\left(p_{j} ; D\right)$ by the minimum principle. We now put $B=\bigcap_{j=1}^{\infty} B_{j}$, so that $B$ is a Borel superset of $Z$ and $\mu_{p_{j}}^{D}(B)=0$ for all $j$. By [10, Corollary 4.7], $\chi_{B}$ is resolutive for $D$ and

$$
S_{\chi_{B}}^{D}(p)=\int_{\partial_{e} D} \chi_{B} d \mu_{p}^{D}=\mu_{p}^{D}(B)
$$

for all $p \in D$. Thus $S_{\chi_{B}}^{D}\left(p_{j}\right)=0$ for all $j$, and so the minimum principle implies that $S_{\chi_{B}}^{D}=0$ on $\bigcup_{j=1}^{\infty} \Lambda\left(p_{j} ; D\right)=D$. Hence $B$ is caloric measure null for $D$.

\section{Dependence on the Open Set}

Theorem 5 below is a partial analogue of $[\mathbf{1}$, Theorem 6.6.10]. In part (b) we assume that $E$ is quasi-regular, a hypothesis not necessary in the harmonic case because there every open set has this property. Thus, as is often the case, the situation for the heat equation is more complicated and less satisfactory than that for Laplace's equation. However, the hypothesis of quasi-regularity is not very restrictive, and examples of open sets which do not satisfy it are always non-trivial. Some examples can be found in [8].

Theorem 5. Let $D$ and $E$ be open sets such that $D \subseteq E$, and let $Z \subseteq \partial_{e} D \cap \partial_{e} E$.

(a) If $Z$ is caloric measure null for $E$, then it is also caloric measure null for $D$.

(b) Conversely, if $E$ is quasi-regular, $Z \subseteq \partial_{e} E \backslash(\overline{E \backslash D})$, and $Z$ is caloric measure null for $D$, then it is also caloric measure null for $E$.

Proof. (a) If $Z$ is caloric measure null for $E$, then for all $q \in E$ the set $Z$ is $\mu_{q}^{E}$-measurable with $\mu_{q}^{E}(Z)=0$. Therefore, by [12, Theorem 10], for every point $q \in D$, the set $Z$ is $\mu_{q}^{D}$-measurable with $0 \leq \mu_{q}^{D}(Z) \leq \mu_{q}^{E}(Z)=0$. Hence $Z$ is caloric measure null for $D$.

(b) For the converse, if $Z$ is contained in the relatively open subset $\partial_{e} E \backslash(\overline{E \backslash D})$ of $\partial_{e} E$, we can write $Z=\bigcup_{i=1}^{\infty} Z_{i}$, where each $Z_{i}$ is bounded and has its closure in 
$\partial_{e} E \backslash(\overline{E \backslash D})$. If we prove that each $Z_{i}$ is caloric measure null for $E$, the result for $Z$ will follow from Theorem 3(b). We can therefore assume that $Z$ is bounded and that $\bar{Z} \subseteq \partial_{e} E \backslash(\overline{E \backslash D})$. Furthermore, if $Z$ is caloric measure null for $D$, Lemma 4 shows that there is a Borel set $B$ such that $Z \subseteq B \subseteq \partial_{e} D$ and which is also caloric measure null for $D$. The set $X=B \cap\left(\partial_{e} E \backslash(\overline{E \backslash D})\right)$ is also a Borel superset of $Z$, and satisfies the same conditions as $Z$. If we were to prove the result with $Z$ replaced by $X$, then the result for $Z$ would follow immediately. We may therefore assume that $Z$ is a Borel set. With this assumption, the inequalities $0 \leq L_{\chi Z}^{E} \leq U_{\chi Z}^{E} \leq 1$ on $E$ imply that $\chi_{Z}$ is resolutive for $E$, by [10, Corollary 4.7].

We now define

$$
w=\left\{\begin{array}{lll}
\chi_{Z} & \text { on } & \partial E, \\
S_{\chi_{Z}}^{E} & \text { on } & E .
\end{array}\right.
$$

Then

$$
\lim _{p \rightarrow q, p \in E} w(p)=0
$$

for every point $q \in \partial_{n} E \backslash \bar{Z}$ that is regular for $E$, and

$$
\lim _{p \rightarrow q+, p \in E} w(p)=0
$$

for every point $q \in \partial_{s s} E \backslash \bar{Z}$ that is regular for $E$. Hence either (1) or (2) holds, as appropriate, for all $q \in \partial_{e} E \backslash \bar{Z}$ outside some polar set, since $E$ is quasi-regular by hypothesis. Moreover, for all $p \in D$ we have $w(p)=S_{\chi Z}^{E}(p)=S_{w}^{D}(p)$, in view of [12, Theorem 11, Corollary].

We now split $\partial_{e} D$ into three disjoint sets, and write

$$
U=\partial_{e} D \cap E \subseteq E \backslash D, \quad V=\partial_{e} D \cap \partial_{e} E \supseteq \bar{Z}, \quad Y=\partial_{e} D \cap \partial_{s} E .
$$

Since $\bar{Z} \subseteq \partial_{e} E$ we have $w=0$ on $\partial_{s} E$, so that

$$
w=w\left(\chi_{U}+\chi_{V}+\chi_{Y}\right)=w \chi_{U}+\chi_{Z}
$$

on $\partial_{e} D$. Therefore, for all $p \in D$ we have

$$
w(p)=S_{w}^{D}(p)=S_{w \chi_{U}}^{D}(p)+S_{\chi_{Z}}^{D}(p)=S_{w \chi_{U}}^{D}(p),
$$

because $Z$ is caloric measure null for $D$. Furthermore, because the compact set $\bar{Z}$ does not meet $\overline{E \backslash D}$, there is a positive distance between them. It follows that, for every point $q \in \bar{Z} \cap \partial_{n} D$ that is regular for $D$,

$$
\lim _{p \rightarrow q, p \in E} w(p)=\lim _{p \rightarrow q, p \in D} w(p)=\lim _{p \rightarrow q} S_{w \chi_{U}}^{D}(p)=0
$$

since $\bar{Z} \cap U=\emptyset$, and for every point $q \in \bar{Z} \cap \partial_{s s} D$ that is regular for $D$,

$$
\lim _{p \rightarrow q+, p \in E} w(p)=\lim _{p \rightarrow q+, p \in D} w(p)=\lim _{p \rightarrow q+} S_{w \chi_{U}}^{D}(p)=0 .
$$

We know from [11, Corollary 8.47] that any point of $\partial_{n} D \cap \partial_{n} E$, or of $\partial_{s s} D(\subseteq$ $\left.\partial_{s s} E\right)$, that is regular for $E$ is also regular for $D$. Moreover, there are no points in $\partial_{n} D \cap \partial_{s s} E$ outside $\overline{E \backslash D}$; for if $q \in \partial_{s s} E$ there is a half-ball $H\left(q, r_{0}\right) \subseteq E$, so that if $q \in \partial_{n} D$ and $r \leq r_{0}$ then $H(q, r) \backslash D \neq \emptyset$, and hence $q \in \overline{E \backslash D}$. Since $E$ is quasi-regular, it now follows that either (3) or (4) holds, as appropriate, for all $q \in \bar{Z}$ outside some polar set. Therefore either (1) or (2) holds, as appropriate, for all $q \in \partial_{e} E$ outside some polar set. If $E$ is unbounded then the point at infinity is regular, by $[\mathbf{1 1}$, Theorem $8.46(\mathrm{~b})]$, and since $Z$ is bounded we have $\chi_{Z}=0$ in a 
neighbourhood of infinity, so that $w(p) \rightarrow 0$ as $p$ approaches infinity also. It now follows from the boundedness of $w$ and the maximum principle in $[\mathbf{1 1}$, Theorem 8.2], that $w=0$ on $E$. Hence $Z$ is caloric measure null for $E$.

\section{Polarity}

We know from $[\mathbf{1 0}, \mathrm{p} .405]$ that any polar subset of $\partial_{e} D$ is caloric measure null for $D$. In this section we show that a subset $Z$ of $\partial_{a}^{*} D$ is caloric measure null for $D$ only if it is polar. The following lemma plays a crucial role in this.

Lemma 6. Let $D$ be an open set, let $c \in \mathbb{R}$, and let $\left.E_{c}=\mathbb{R}^{n} \times\right] c,+\infty[$. If

$$
Z \subseteq\left(\partial_{e} D \cap\left(\mathbb{R}^{n} \times\{c\}\right)\right) \backslash\left(\overline{E_{c} \backslash D}\right),
$$

then $Z$ is caloric measure null for $D$ only if $Z$ is polar.

Proof. Suppose that $Z$ is caloric measure null for $D$. We first consider the case where $D \subseteq E_{c}$. Since $E_{c}$ is a regular open set and (5) holds, it follows from Theorem 5 (b) that $Z$ is caloric measure null for $E_{c}$. The caloric measure of $Z$ relative to $E_{c}$ and any point $(x, t)$ therein, is given by

$$
\mu_{(x, t)}^{E_{c}}(Z)=\int_{Z} W(x-y, t-c) d y
$$

[2, p. 332], which is zero if and only if the $n$-dimensional Lebesgue measure of $Z$ is zero. By [11, Theorem 7.55], this happens if and only if the thermal capacity of $Z$ relative to $\mathbb{R}^{n+1}$ is zero, which holds if and only if $Z$ is polar [11, Theorem 7.46].

We now consider the general case, and put $C=D \cap E_{c}$. We note that condition (5) implies that $Z \subseteq \partial_{n} C$; for if $q \in \partial_{e} D \cap\left(\mathbb{R}^{n} \times\{c\}\right)$, then either (i) for all $r>0$ we have $H^{*}(q, r) \cap D \neq \emptyset$, in which case $q \in \partial_{n} C$, or (ii) there is $\epsilon>0$ such that $H^{*}(q, \epsilon) \cap D=\emptyset$, in which case $q \in \overline{E_{c} \backslash D}$. Thus $Z \subseteq \partial_{e} C \cap \partial_{e} D$, and we can use Theorem 5(a) to show that $Z$ is caloric measure null for $C$. Since $E_{c} \backslash C=E_{c} \backslash D$, condition (5) holds with $D$ replaced by $C$ throughout. Therefore we can use the case proved above to show that $Z$ is polar.

Lemma 7. If $A$ is an analytic set whose every compact subset is polar, then $A$ itself is polar.

Proof. Let $K$ be any compact subset of $A$. Since $K$ is polar it has thermal capacity $\mathcal{C}(K)=0[\mathbf{1 1}, \mathrm{p} .175]$. Therefore the inner thermal capacity of $A$, namely $[\mathbf{1 1}$, Definition 7.40]

$$
\mathcal{C}_{-}(A)=\sup \{\mathcal{C}(K): K \text { is a compact subset of } A\},
$$

is also zero. Since $A$ is analytic it is thermal capacitable, by [11, Theorem 7.51], with thermal capacity $\mathcal{C}(A)=\mathcal{C}_{-}(A)=0[\mathbf{1 1}$, Definition 7.43]. Therefore $A$ is polar, by $[\mathbf{1 1}$, Theorem $7.46(\mathrm{~b})]$.

Theorem 8. Let $D$ be an open set. A subset $Z$ of $\partial_{a}^{*} D$ is caloric measure null for $D$ if and only if it is polar.

Proof. Suppose that $Z$ is caloric measure null for $D$. By Lemma 4, there is a Borel subset $B$ of $\partial_{e} D$ which contains $Z$ and is also caloric measure null for $D$. The set $B \cap \partial_{a}^{*} D$ is a Borel set which contains $Z$ and satisfies the same conditions as $Z$. We can therefore suppose that $Z$ is Borel. 
By [11, Theorem 8.40], there is a sequence of hyperplanes of the form $\mathbb{R}^{n} \times\{t\}$ which covers $\partial_{a}^{*} D$. We can therefore write $Z=\bigcup_{i=1}^{\infty} Z_{i}$, where each $Z_{i}$ is either $\emptyset$ or a caloric measure null subset of a single such hyperplane. It thus suffices to prove that any nonempty $Z_{i}$ is polar. So we suppose that $\emptyset \neq Z_{i} \subseteq \mathbb{R}^{n} \times\{c\}$, and take an arbitrary compact subset $K$ of $Z_{i}$. We put $\left.E_{c}=\mathbb{R}^{n} \times\right] c,+\infty[$ with a view to using Lemma 6 . For each point $q \in K$, we can find $r_{q}>0$ such that $H^{*}\left(q, r_{q}\right) \subseteq D$ because $q \in \partial_{a}^{*} D$, and also $B\left(q, r_{q}\right) \cap\left(\mathbb{R}^{n} \times\{c\}\right) \subseteq \partial_{a}^{*} D$ because $\partial_{a}^{*} D$ is relatively open in $\partial D$. The compactness of $K$ ensures that there is $\epsilon>0$ such that $H^{*}(q, \epsilon) \subseteq D$ and $B(q, \epsilon) \cap\left(\mathbb{R}^{n} \times\{c\}\right) \subseteq \partial_{a}^{*} D$ for all $q \in K$. Thus the distance between $K$ and $E_{c} \backslash D$ is positive, so that $K \cap\left(\overline{E_{c} \backslash D}\right)=\emptyset$. Hence $K \subseteq \partial_{e} D \cap\left(\mathbb{R}^{n} \times\{c\}\right) \backslash\left(\overline{E_{c} \backslash D}\right)$, and Lemma 6 shows that $K$ is polar. If $Z$ is a Borel set then each $Z_{i}$ is too, so that $Z_{i}$ is analytic by [11, Lemma 7.49(b)]. Hence the arbitrariness of $K$ and Lemma 7 show that $Z_{i}$ is polar.

\section{References}

[1] D. H. Armitage and S. J. Gardiner, Classical Potential Theory, SpringerVerlag, London, 2001.

[2] J. L. Doob, Classical Potential Theory and its Probabilistic Counterpart, Grundlehren der mathematischen Wissenschaften 262, Springer-Verlag, New York, 1984.

[3] E. Fabes and S. Salsa, Estimates of caloric measure and the initial-Dirichlet problem for the heat equation in Lipschitz cylinders, Trans. Amer. Math. Soc. 279 (1983), 635-650.

[4] Y. Heurteaux, Mésure harmonique et l'équation de la chaleur, Ark. Mat. 34 (1996), 119-139.

[5] R. Kaufman and J. Wu, Parabolic potential theory, J. Differ. Equations 43 (1982), 204-234.

[6] R. Kaufman and J.-M. Wu, Dirichlet problem of heat equation for $C^{2}$ domains, J. Differ. Equations 80 (1989), 14-31.

[7] J. L. Lewis and J. Silver, Parabolic measure and the Dirichlet problem for the heat equation in two dimensions, Indiana Univ. Math. J. 37 (4) (1988), 801-839.

[8] S. J. Taylor and N. A. Watson, A Hausdorff measure classification of polar sets for the heat equation, Math. Proc. Camb. Philos. Soc. 97 (1985), 325-344.

[9] N. A. Watson, Green functions, potentials, and the Dirichlet problem for the heat equation, Proc. Lond. Math. Soc. Ser. III 33 (1976), 251-298.

[10] N. A. Watson, Caloric measure for arbitrary open sets, J. Aust. Math. Soc. 92 (3) (2012), 391-407.

[11] N. A. Watson, Introduction to Heat Potential Theory, Mathematical Surveys and Monographs 182, Amer. Math. Soc., Providence, 2012.

[12] N. A. Watson, The two versions of the Dirichlet problem for the heat equation, New Zealand J. Math. 45 (2015), 89-110.

[13] N. A. Watson, Uniqueness of extendable temperatures, Bull. Aust. Math. Soc. 103 (2) (2021), 311-317.

[14] J.-M. G. Wu, On parabolic measures and subparabolic functions, Trans. Amer. Math. Soc. 251 (1979), 171-185; Erratum, ibidem 259 (1980), 636. 
[15] J.-M. Wu, An example on null sets of parabolic measures, Proc. Amer. Math. Soc. 107 (4) (1989), 949-961.

Neil A. Watson

School of Mathematics and Statistics,

University of Canterbury,

Private Bag 4800, Christchurch,

New Zealand 8140

neil.watson@canterbury.ac.nz 